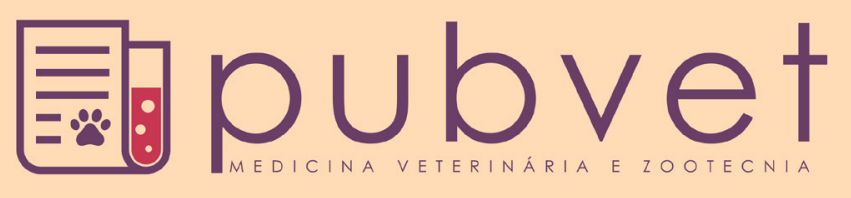

HTTP://DX.DOI.ORG/10.22256/PUBVET.V11N4.393-398

\title{
Morfometria e craniometria de Tuim-de-asa-azul (Forpus xanthopterygius Spix, 1824)
}

\author{
Joyce Galvão de Souza ${ }^{1 *}$, Artur da Nóbrega Carreiro ${ }^{2}$, Ana Yasha Ferreira de La Salles ${ }^{2}$, \\ Moana Barbosa dos Santos Figuerêdo', Stephenson Hallison Formiga Abrantes ${ }^{3}$, José \\ Rômulo Soares dos Santos ${ }^{4}$, Danilo José Ayres de Menezes ${ }^{5}$
}

${ }^{1}$ Aluna de Graduação em Medicina Veterinária, Universidade Federal de Campina Grande, Unidade Acadêmica de Medicina Veterinária, Patos, Paraiba, Brasil.E-mail: joycegalvaosouza@gmail.com; moana_figueredo@hotmail.com

${ }^{2}$ Aluno de Mestrado do Programa de Pós-graduação em Medicina Veterinária, Universidade Federal de Campina Grande, Unidade Acadêmica de Medicina Veterinária, Patos, Paraíba,Brasil.E-mail: arturpets@gmail.com; anayasha@hotmail.com

${ }^{3}$ Professor da Unidade Acadêmica de Ciências Biológicas da Universidade Federal de Campina Grande, Patos, Paraiba, Brasil. E-mail: stephensonabrantes@yahoo.com.br

${ }^{4}$ Doutor em Medicina Veterinária da Universidade Federal de Campina Grande, Unidade Acadêmica de Medicina Veterinária, Patos, Paraiba, Brasil.E-mail:jromulosmedvet@hotmail.com

${ }^{5}$ Professor do Departamento de Morfologia da Universidade Federal do Rio Grande do Norte, Natal, Brasil. Email: mdanayres@gmail.com *Autor de correspondência

RESUMO. O estudo Craniométrico e Prosoprométrico é de grande valia para se estabelecer dados que podem vir a tornar-se chave para identificação da espécie. São escassos na literatura dados morfométricos e descritivos de crânios de psitacídeos. O presente estudo buscou descrever os principais ossos e estabelecer medidas do crânio de "Tuim-de-asa-azul" (Forpus xanthopterygius). Foram utilizadas cabeças de exemplares adultos, um macho e uma fêmea, doados pelo Centro de Triagem de Animais Silvestres da Paraíba (CETAS - PB). Estas foram submetidas a maceração e à identificação, caracterização e medição das estruturas e dos ossos componentes de seus respectivos crânios. Apesar do processo natural de fusionamento dos ossos dificultar a identificação dos mesmos, foi possível identificar os ossos frontal, quadrado, arco jugal, maxilar e mandíbula. Estes ossos são característicos e facilmente identificados, servindo como base para localização e identificação dos demais. O comprimento máximo do crânio foi de 29,0 $\mathrm{mm}$ e a largura de $16,0 \mathrm{~mm}$. Os dados obtidos servem de base para a caracterização taxonômica da espécie, já que não são encontrados dados na literatura.

Palabras chave: anatomía, aves, psittacidae

\section{Morphometry and craniometry of Blue-winged parrotlet (Forpus xanthopterygius)}

\begin{abstract}
The Craniometric and Prosoprometric study is of great value to establish data that may become a key to identification of the species. Morphometric and descriptive data of psittacine skulls are scarce in the literature. The present study aimed to describe the main bones and to establish measures of the skull of "Blue-winged parrotlet" (Forpus xanthopterygius). Two heads of adult specimens, one male and one female, were donated by the Paraíba Wild Animals Triage Center (CETAS - PB). These were subjected to maceration and to the identification, characterization and measurement of structures and component bones of their respective skulls. Although the natural process of fusing the bones made it difficult to identify the bones, it was possible to identify the frontal, square, jugal, maxillary and mandible bones. These bones are characteristic and easily identified, serving as a basis for locating and identifying others. The maximum length of the skull was
\end{abstract}


$29.0 \mathrm{~mm}$ and the width of $16.0 \mathrm{~mm}$. The data obtained serve as a basis for the taxonomic characterization of the species, since no data are found in the literature.

Keywords: anatomy, birds; psittacidae

\section{Morfometría y craneometría de Periquito de ala azul (Forpus xanthopterygius Spix, 1824)}

RESUMEN. El estudio Craniométrico y Prosoprométrico es de gran valor para establecer los datos que pueden convertirse en clave para la identificación de especies. En la literatura hay pocos datos morfométricos y descriptivos de cráneos de periquitos. Este estudio tuvo como objetivo describir los principales huesos del cráneo y establecer medidas del "Periquito de ala azul" (Forpus xanthopterygius). Se utilizaron cabezas de animales adultos, un macho y una hembra, donados por el Centro de Triagem de Animais Silvestres de Paraíba (CETAS - PB). Estas se sometieron a maceración y a la identificación, caracterización y medición de estructuras óseas de los respectivos cráneos. A pesar del proceso natural de fusión de los huesos dificultar la identificación de los mismos, se pudo identificar el hueso frontal, cuadrado, arco malar, maxilar y mandíbula. Estos huesos son característicos y fácilmente identificados, sirviendo como base para la localización e identificación de los demás. La longitud máxima del cráneo fue $29,0 \mathrm{~mm}$ y la anchura de $16.0 \mathrm{~mm}$. Los datos pueden servir como base para la caracterización taxonómica de la especies, ya que no son encentrados en la literatura.

Palabras clave: anatomía, Catita enana, pájaros, psitácidas

\section{Introdução}

O gênero Forpus é composto por sete espécies, e estes são encontrados em toda a região neotropical (Forshaw and Cooper, 1989). O Forpus xanthopterygius é o menor dos psitacídeos presentes no Brasil e possuem dimorfismo sexual por característica externa, não muito encontrada nos demais exemplares da ordem (Cubas et al., 2014), sendo caracterizada pela coloração azul na asa e na região do dorso caudal do macho, com o restante do corpo verde, enquanto que a fêmea é totalmente verde com áreas amareladas (LyraNeves and Telino-Júnior, 2010).

Desde o século XIX o estudo do crânio gera dados que vem contribuindo para uma melhor identificação das aves, o que possibilita a classificação e estabelecimento de similaridades entre diferentes táxons (Pascotto et al., 2006) e para entendimento do processo evolutivo através da identificação de suas particularidades e funções (Caldas, 2015).

Por outro lado, para o correto tratamento de lesões ósseas, se faz necessário o conhecimento das estruturas e particularidades dos ossos de cada espécie, além de auxiliar no estudo dos demais sistemas dos organismos (Caldas, 2015). O crânio das aves apresenta muitas variações dentro da mesma família ou ordem, sendo que estas particularidades demonstram adaptações próprias de acordo com os hábitos alimentares da espécie (Pascotto et al., 2006).

Os dados obtidos em pesquisas que são desenvolvidas com espécies de nossa fauna contribuem diretamente para $\mathrm{o}$ melhor atendimento e suporte às mesmas. Existem poucos estudos que definem as particularidades anatômicas e morfométricas do crânio de psitacídeos, portanto, pretendeu-se estabelecer valores craniométricos e prosoprométricos de dois exemplares de Tuim-de-asa-azul (Forpus xanthopterygius) identificando os principais ossos e estruturas a fim de auxiliar tanto os biólogos, zoólogos e taxonomistas, bem como outros profissionais da área, assim como a médicos veterinários no conhecimento e identificação das particularidades anatômicas para a realização de procedimentos clínicos e cirúrgicos referentes a espécie.

\section{Material e Métodos}

Foram utilizados dois cadáveres de Tuins-deasa-azul (Forpus xanthopterygius), um macho e uma fêmea, doados pelo Centro de Triagem de Animais Silvestres da Paraíba (CETAS-PB). O estudo foi conduzido no Laboratório de Pesquisas Morfológicas da Unidade Acadêmica de Medicina Veterinária (UAMV), Centro de Saúde e Tecnologia Rural (CSTR), da Universidade Federal de Campina Grande (UFCG), Patos/PB. 
Foi realizada maceração controlada dos crânios, iniciando com rebatimento da pele, fáscias e musculatura, com instrumental de dissecação. Em seguida os crânios foram submetidos à ação de insetos da espécie Solenopsis spp com a finalidade de preservar as junções ósseas, processo adaptado de Ladeira and Höfling (2007). Após completo consumo das partes moles do crânio pelos insetos, procedeu-se com a clarificação com peróxido de hidrogênio a 5\% (Ladeira and Höfling, 2007) durante 5 minutos e uma nova limpeza dos tecidos finalizou a preparação dos crânios.

A descrição dos ossos e obtenção dos valores craniométricos e prosoprométricos foi obtida com o auxílio de lupa circular de luz fria. As mensurações foram realizadas utilizando como referências mensurações utilizadas por Werner et al. (2015) com adaptações e com auxílio de paquímetro universal de $0,00 \mathrm{~mm}$. Foi mensurado o comprimento máximo do crânio, medido do ponto mais caudal do crânio, logo acima da proeminência cerebelar até a face rostral da rinoteca, seguindo uma linha horizontal; a largura máxima do crânio foi obtida de um processo pós orbital ao outro contra lateral; a largura caudal máxima do crânio foi obtida da região dorsal ao meato acústico externo de um antímero até o seu contra lateral; a distância dos processos para- occipitais foi obtida entre seus pontos mais mediais; diâmetro e largura do forame magno; comprimento e espessura do arco jugal; altura da ranfoteca; largura máxima da rinoteca; largura máxima da gnatoteca; largura da zona flexora crânio facial e distância entre os ossos lacrimais.

\section{Resultados e Discussão}

Foi observado que os ossos do crânio dos Tuins adultos tendem a se fusionar com a idade, assim como relatado em outras espécies de aves (Brito, 2008, Guzzi et al., 2014, Previatto, 2012, Cavinatto et al., 2016) apesar de tal processo, foi possível identificar alguns ossos como: frontal, quadrado, arco jugal, pterigoide, vômer, rostroparaesfenoidal, maxila, mandíbula, parietal, occipital, lacrimal, palatino, nasal, esfenoide e supraoccipital; além de algumas estruturas como a proeminência cerebelar, o forame óptico, o processo pós-orbital, zona flexora craniofacial, septo interorbital, lâmina paraesfenoidal, arco suborbital, fontículo orbitocranial, meato acústico externo, crista nucal transversa, processo paraoccipital, fossa subcondilar, fenestra rostral da mandíbula, vômer, exoccipital, côndilo occipital e forame magno (Figura 1). As mensurações obtidas dos crânios estão presentes na Tabela 1.

Tabela 1. Medições Craniométricas e Prosoprométricas de Tuim-de-asa-azul (Forpus xhantopterygius)

\begin{tabular}{lccc}
\hline Medições, crânio & Fêmea, mm & Macho, mm & Média, mm \\
\hline Comprimento máximo do crânio & 29,8 & 28,2 & 29 \\
Largura máxima do crânio & 16,0 & 16,0 & 16 \\
Largura caudal máxima do crânio & 15,8 & 16,0 & 15,9 \\
Distância dos processos para-occipitais & 9,8 & 10,0 & 9,9 \\
Diâmetro do forame magno & 4,0 & 4,2 & 4,1 \\
Largura do forame magno & 2,9 & 2,9 & 2,9 \\
Comprimento do arco jugal & 12,9 & 13,1 & 13 \\
Espessura do arco jugal & 0,09 & 0,09 & 0,09 \\
Altura máxima da ranfoteca & 13,1 & 13,8 & 13,45 \\
Largura máxima da rinoteca & 8,0 & 8,8 & 8,4 \\
Largura máxima da gnatoteca & 8,1 & 8,1 & 8,1 \\
Largura da zona flexora craniofacial & 9,0 & 8,5 & 8,75 \\
Distância entre os ossos lacrimais & 12,1 & 12,4 & 12,25 \\
\hline
\end{tabular}

Assim como descrito por Cavinatto et al. (2016), em estudo realizado com exemplares do gênero Amazona, constatou-se cavidades orbitárias capazes de acomodar grandes globos oculares e uma zona flexora craniofacial bem desenvolvida, o que também vai de acordo com Cubas et al. (2014) que afirmam serem estas características comuns à ordem Psitaciformes.
O espaço entre as órbitas apresentou um padrão achatado denominado de septo interorbital e a zona flexora crânio facial se apresentou bem demarcada, entre os ossos nasal e frontal, possibilitando amplo movimento em sentidos dorsal e ventral da rinoteca, o que se assemelha ao descrito por Cavinatto et al. (2016) para outras espécies da mesma ordem. 
Os ossos quadrados foram identificados como sendo de formato quase triangular articulando-se com o arco jugal, osso pterigoide e mandíbula. Além dessas conexões, não foi possível identificar com segurança mais estruturas como as descritas por Guzzi et al. (2014) (osso quadrático, processo orbital do quadrado e processo ótico do quadrado) devido ao reduzido tamanho do osso quadrado de
Tuim-de-asa-azul. Identificou-se a fenestra rostral no terço cranial da mandíbula, o que difere do encontrado por Posso and Donatelli (2007) para exemplares da subfamília Coccizynae, que não apresentam fenestra rostral da mandíbula, apenas fenestra caudal, a qual não foi identificada nos exemplares deste estudo.
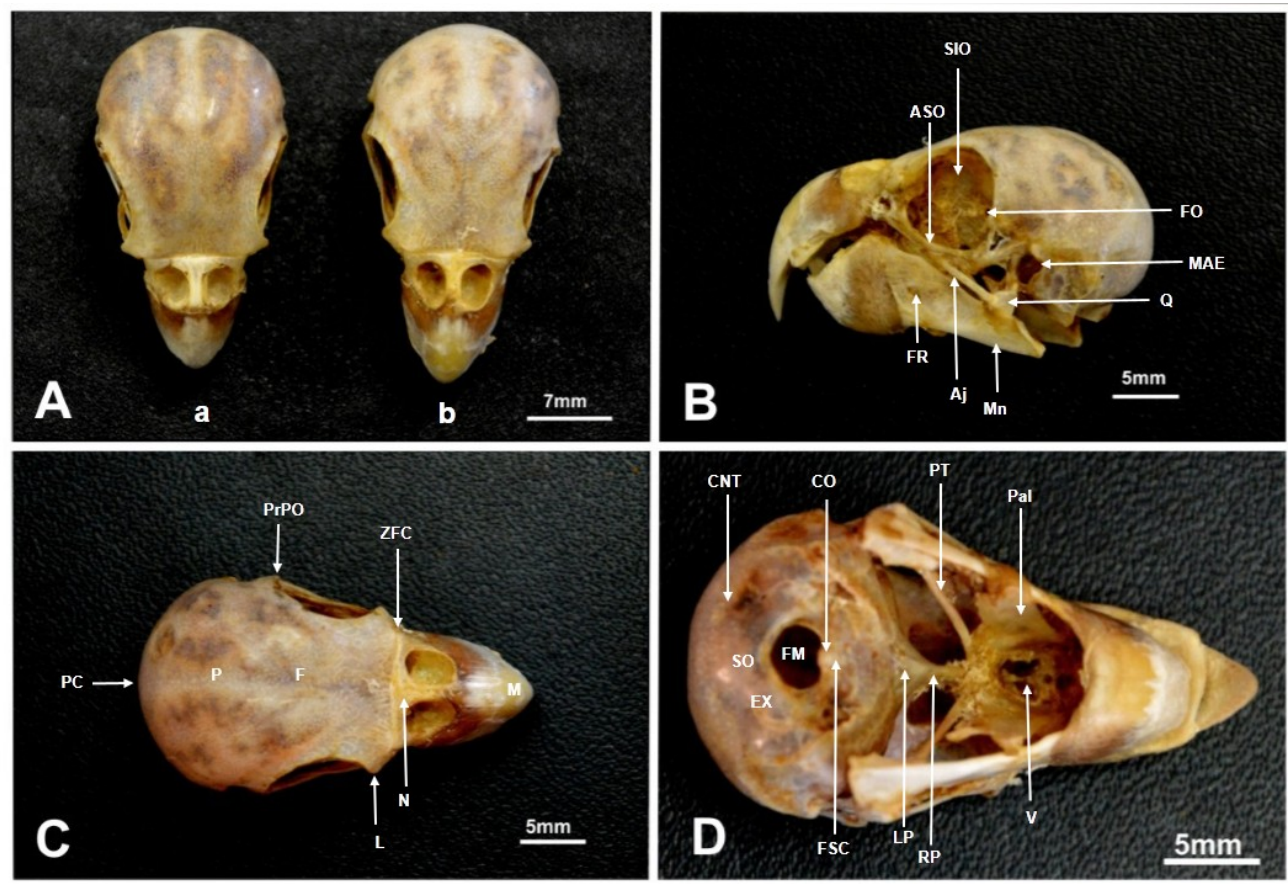

Figura 1 A-D: A: Vista dorsal dos crânios do macho (a) e fềmea (b); B: Vista lateral do crânio; C: Vista dorsal do crânio; D: Vista ventral do crânio. Aj: arco jugal; ASo: arco suborbital; CO: côndilo do occipital; CNT: crista nucal transversa; EX: exoccipital; FR: fenestra rostral da mandíbula; FM: forame magno; FO: forame óptico; FSC: fossa subcondilar; F: frontal; L: lacrimal; LP: lâmina paraesfenoidal; Mn: mandíbula; Mx: maxila; MAE: meato acústico externo; N: nasal; Pal: palatino; P: parietal; PrPO: processo pós-orbital; PC: proeminência cerebelar; PT: pterigoide; Q: quadrado; RP: rostro paraesfenoidal; SIO: septo interorbital; SO: supra-occipital; V: vômer; ZFC: zona flexora craniofacial.

A região frontal rostralmente se articula com a zona flexora craniofacial bem pronunciada, caudalmente se funde ao osso parietal, sem sutura visível, e ventralmente se funde com o septo interorbital, enquanto que laterorostralmente se articula com os ossos lacrimais sem sutura visível entre eles; diferente do que foi observado por Posso and Donatelli (2007) em que a sutura lacrimofrontal foi identificada entre o osso lacrimal e a região frontal, provavelmente por serem animais mais jovens que os observados neste estudo. Ainda, segundo Posso and Donatelli (2007), a região parietal dos Coccizynae se apresentou completamente fundida à região frontal, e o mesmo foi observado no crânio de Tuim-de-asa-azul, o que dificultou uma delimitação clara entre as duas regiões.

$\mathrm{O}$ osso pterigoide, juntamente com os ossos palatinos e o vômer, forma a região do palato
(Guzzi et al., 2014). Nos exemplares estudados, os ossos pterigoides foram facilmente identificados e mensurados, obtendo-se valor médio de $8,3 \mathrm{~mm}$ na fêmea e $8,05 \mathrm{~mm}$ no macho (Tabela 1). $\mathrm{O}$ rostroparaesfenoidal apresentou-se afilado, principalmente em sua porção mais rostral, semelhante ao descrito por Flausino Júnior et al. (1999) para exemplares de Momotus momota, e o formato foi levemente côncavo. A lâmina paraesfenoidal se apresentou inclinada no sentido medial e dorsal do interior do crânio, terminando em uma ponta afilada que está acima da porção inicial do rostroparaesfenoidal. $\mathrm{O}$ vômer pôde ser identificado como uma estrutura estreita e achatada lateralmente; o que se assemelha ao descrito por Guzzi et al. (2014) para exemplares de Caracara cheriway e Caracara plancus, porém, nestes exemplares o vômer se apresentou como uma lâmina longa, enquanto que nos Tuins- 
de-asa-azul teve comprimento relativamente curto.

O osso lacrimal é uma projeção afilada e cranial na órbita, que encerra a margem orbital, destacando a região da órbita com forma proeminente que se estende até o processo pósorbital, a qual segue a borda lateral do osso frontal em sua região frontal e dorsal, e do osso parietal em sua região mais caudal, sendo que a sutura entre a região frontal e o osso lacrimal não são visíveis, porém a sutura lacrimonasal, entre o osso lacrimal e o nasal é visível e bem delimitada, se assemelhando ao descrito em Piaya cayana (Posso and Donatelli, 2007). O arco suborbital, que resulta da fusão do processo pós-orbital e do processo zigomático com o processo lacrimal, mostrou-se completo e com semelhança ao descrito para o gênero Nymphicus (Gaban-Lima, 2007). O fontículo orbitocranial foi facilmente identificado caudodorsal ao esfenoide, com abertura em sentido rostral, enquanto que o forame óptico se abre em sentido rostrolateral e localizase caudoventral às bordas ventral e caudal do fontículo orbitocranial, diferente do que foi descrito para Gymnogyps amplus (Brito, 2008) onde o fontículo interorbital e o forame óptico são fusionados.

O meato acústico externo tem formato aproximado de círculo, na altura da região ventral da órbita e dorsocaudal ao osso quadrado, sendo assim, entre os exemplares estudados por (GabanLima, 2007), mais semelhante à Cyanoliseus.

A crista da nuca transversa mostrou-se pouco desenvolvida em sua porção dorsal e mais desenvolvida em suas porções laterais, apresentando semelhança à Aratinga aurea, (Gaban-Lima, 2007). A proeminência cerebelar mostrou-se reduzida, semelhante ao descrito por Pascotto et al. (2006) para exemplares de Bucorvus cafer, porém, delimitada dorsalmente pela crista nucal transversa. O osso supraoccipital é proeminente e lateralmente à ele, à direita e à esquerda, estavam também bem demarcados os exoccipitais, o que confere com a organização descrita por Posso and Donatelli (2007) para a subfamília Coccyzinae. O processo paraoccipital em ambos exemplares se mostrou longos e afilados, com curvatura em sentido medial na sua porção mais ventral, que se assemelha ao descrito por Migotto (2008) para espécie Gyps fulvus.

A fossa subcondilar apresentou-se como uma estrutura rasa, semelhante ao encontrado para Rosthramus sociabilis (Migotto, 2008). Acima da fossa subcondilar o forame magno, que teve comprimento médio de $4,1 \mathrm{~mm}$ e largura média de $2,9 \mathrm{~mm}$, apresentou-se direcionado ventralmente. Estes dados demonstram medidas e particularidades de indivíduos adultos, entretanto, para que possamos extrapolar os achados à espécie, o número de animais deverá ser aumentado para estabelecimento da média das medidas analisadas

A fusão dos ossos em Tuins adultos dificulta a identificação de suas divisões, sendo possível identificar claramente apenas ossos como frontal, quadrado, arco jugal, vômer, nasal, maxilar, mandibular, lacrimal e esfenoide necessitando de instrumentos de aumento para melhor visualização e delimitação. Os dados morfométricos obtidos, apesar de serem em apenas um exemplar de macho e outro de fêmea, são bases que podem servir para a caracterização taxonômica para a espécie, já que não são encontrados dados na literatura.

\section{Referências Bibliográficas}

Brito, G. R. R. 2008. Análise filogenética de Cathartidae (Aves) com base em caracteres osteológicos. Universidade de São Paulo, São Paulo, Brasil.

Caldas, S. S. 2015. Estudo morfológico descritivo das estruturas relacionadas à cinética craniana do Ramphastos toco. Faculdade de Agronomia e Medicina Veterinária. Universidade de Brasília, Brasília.

Cavinatto, C. C., Armando, A. P. R. N., Cruz, L. K. S., Lima, E. M. M. \& Santana, M. I. S. 2016. Descrição anatômica de esqueletos de papagaios do gênero Amazona através da utilização de radiografias. Pesquisa Veterinária Brasileira, 36, 123-130.

Cubas, Z. S., Silva, J. C. R. \& Dias, J. L. C. 2014. Tratado de animais selvagens-medicina veterinária. Editora Roca.

Flausino Júnior, O. A., Donatelli, R. J. \& Pascotto, M. C. 1999. Osteologia e miologia cranianas de Momotus momota (Coraciformes: Momotidae). Ararajuba, 7, 109-124.

Forshaw, J. M. \& Cooper, W. T. 1989. Parrots of the world. Blandford, London.

Gaban-Lima, R. 2007. Análise filogenética de psittaciformes (aves) com base em caracteres morfológicos siringeais e osteológicos. Universidade de São Paulo, São Paulo.

Guzzi, A., Santos Nascimento, M., Lima, S. P., Santos, S. S. \& Donatelli, R. J. 2014. 
Osteologia craniana e aspectos evolutivos de Mycteria (Aves: Ciconiidae). Revista Nordestina de Biologia, 23, 85-103.

Ladeira, L. M. C. E. B. \& Höfling, E. 2007. Osteologia craniana de Bucconidae. Boletim do Museu Paraense Emílio Goeldi Ciências Naturais, 2, 117-153.

Lyra-Neves, R. \& Telino-Júnior, W. 2010. Aves da fazenda Tamanduá. Avis Brasilis Editora, Vinhedo, São Paulo.

Migotto, R. 2008. Inferência filogenética em gaviões buteoninos (Aves: Accipitridae), com base em caracteres osteológicos cranianos. Universidade de São Paulo, São Paulo.

Pascotto, M. C., Höfling, E. \& Donatelli, R. J. 2006. Cranial osteology of Coraciiformes (Aves). Revista Brasileira de Zoologia, 23, 841-864.

Posso, S. R. \& Donatelli, R. J. 2007. Osteologia craniana e considerações sistemáticas em
Coccyzinae (Cuculidae). Boletim do Museu Paraense Emílio Goeldi Ciências Naturais, 2, 87-116.

Previatto, D. M. 2012. Osteologia craniana da família Anhimidae (Aves: Anseriformes).

Werner, L. C., Silva, L. C. S. \& Souza, R. A. M. 2015. Dados craniométricos de Curicacas (Theristicus caudatus). Anais do $42^{\circ}$ Congresso Brasileiro de Medicina Veterinária e $1^{\circ}$ Congresso Sul-Brasileiro da ANCLIVEPA.

\section{Article History:}

Received 25 December 2016

Accepted 7 February 2017

Available on line 23 March 2017

License information: This is an open-access article distributed under the terms of the Creative Commons Attribution License 4.0, which permits unrestricted use, distribution, and reproduction in any medium, provided the original work is properly cited. 